\title{
2D cine DENSE with low encoding frequencies accurately quantifies cardiac mechanics with improved image characteristics
}

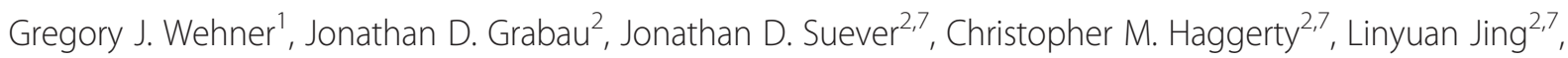
David K. Powell ${ }^{1}$, Sean M. Hamlet ${ }^{3}$, Moriel H. Vandsburger ${ }^{5}$, Xiaodong Zhong ${ }^{4}$ and Brandon K. Fornwalt ${ }^{1,2,5,6,7,8^{*}}$

\begin{abstract}
Background: Displacement Encoding with Stimulated Echoes (DENSE) encodes displacement into the phase of the magnetic resonance signal. The encoding frequency $\left(k_{e}\right)$ maps the measured phase to tissue displacement while the strength of the encoding gradients affects image quality. 2D cine DENSE studies have used a $k_{e}$ of 0.10 cycles/ $\mathrm{mm}$, which is high enough to remove an artifact-generating echo from k-space, provide high sensitivity to tissue displacements, and dephase the blood pool. However, through-plane dephasing can remove the unwanted echo and dephase the blood pool without relying on high $\mathrm{k}_{\mathrm{e}}$. Additionally, the high sensitivity comes with the costs of increased phase wrapping and intra-voxel dephasing. We hypothesized that $k_{e}$ below $0.10 \mathrm{cycles} / \mathrm{mm}$ can be used to improve image characteristics and provide accurate measures of cardiac mechanics.
\end{abstract}

Methods: Spiral cine DENSE images were obtained for 10 healthy subjects and 10 patients with a history of heart disease on a 3 T Siemens Trio. A mid-ventricular short-axis image was acquired with different ke: 0.02, 0.04, 0.06, 0.08 , and 0.10 cycles $/ \mathrm{mm}$. Peak twist, circumferential strain, and radial strain were compared between acquisitions employing different $k_{e}$ using Bland-Altman analyses and coefficients of variation. The percentage of wrapped pixels in the phase images at end-systole was calculated for each $k_{e}$. The dephasing of the blood signal and signal to noise ratio (SNR) were also calculated and compared.

Results: Negligible differences were seen in strains and twist for all $k_{e}$ between 0.04 and 0.10 cycles $/ \mathrm{mm}$. These differences were of the same magnitude as inter-test differences. Specifically, the acquisitions with 0.04 cycles $/ \mathrm{mm}$ accurately quantified cardiac mechanics and had zero phase wrapping. Compared to $0.10 \mathrm{cycles} / \mathrm{mm}$, the acquisitions with 0.04 cycles/mm had $9 \%$ greater SNR and negligible differences in blood pool dephasing.

Conclusions: For 2D cine DENSE with through-plane dephasing, the encoding frequency can be lowered to 0.04 cycles/mm without compromising the quantification of twist or strain. The amount of wrapping can be reduced with this lower value to greatly simplify the input to unwrapping algorithms. The strain and twist results from studies using different encoding frequencies can be directly compared.

Keywords: DENSE, Displacement, Cardiac mechanics, Encoding frequency, Strain, Twist, Magnetic resonance

\footnotetext{
* Correspondence: bkf@gatech.edu

'Department of Biomedical Engineering, University of Kentucky, Lexington, KY, USA

${ }^{2}$ Department of Pediatrics, University of Kentucky, Lexington, KY, USA

Full list of author information is available at the end of the article
}

(c) 2015 Wehner et al. Open Access This article is distributed under the terms of the Creative Commons Attribution 4.0 International License (http://creativecommons.org/licenses/by/4.0/), which permits unrestricted use, distribution, and reproduction in any medium, provided you give appropriate credit to the original author(s) and the source, provide a link to the Creative Commons license, and indicate if changes were made. The Creative Commons Public Domain Dedication waiver (http://creativecommons.org/publicdomain/zero/1.0/) applies to the data made available in this article, unless otherwise stated. 


\section{Background}

Displacement Encoding with Stimulated Echoes (DENSE) is a cardiovascular magnetic resonance (CMR) technique that encodes tissue displacement into the phase of the MR signal [1]. The resulting pixel-level resolution of the displacement field has been used to quantify cardiac mechanics in both healthy and diseased animals and humans [1-6]. The encoding gradient strength is proportional to the displacement sensitivity of the phase images. It is often referred to as the encoding frequency $\left(k_{e}\right)$ with units of cycles $/ \mathrm{mm}$.

In addition to specifying sensitivity, the $\mathrm{k}_{\mathrm{e}}$ plays a role in several other processes related to image quality and postprocessing. The earliest implementations of DENSE relied on a high $\mathrm{k}_{\mathrm{e}}$ to shift the artifact-generating echoes beyond the sampled region of k-space [1] (Fig. 1, column 1). While this technique removed stripe artifacts, the high encoding gradients caused significant intra-voxel dephasing in deforming tissue, which limited the ability to properly encode displacement during systole [1]. The incorporation of complementary spatial modulation of magnetization (CSPAMM) for echo suppression removed the first artifact-generating echo (the T1 relaxation echo) [3] (Fig. 1, column 2). This allowed for lower $k_{e}$, and thus lower gradients leading to less intra-voxel dephasing, since only the furthest echo (the stimulated anti-echo) had to be shifted out of the k-space field of view. Finally, the addition of a thru-plane dephasing gradient selectively dephased the stimulated anti-echo while preserving the desired stimulated echo [7] (Fig. 1, column 4). This final addition removed the dependence on high $\mathrm{k}_{\mathrm{e}}$ for artifact suppression.

A low $k_{\mathrm{e}}$ is desired to improve the signal to noise ratio (SNR) by reducing the amount of intra-voxel dephasing and to prevent excessive wrapping in the phase images. Recent studies with 2D DENSE have used an in-plane $k_{e}$ of 0.10 cycles $/ \mathrm{mm}$, which creates wrapping in most subjects as only $5 \mathrm{~mm}$ of displacement is required before wrapping occurs $[5,8,9]$. Unwrapping algorithms have been developed and utilized, but they are not guaranteed to be error-free in all subjects or all regions of a given subject's heart [10]. Regions with high velocities and noise are the most challenging for automated and semiautomated techniques. Importantly, DENSE studies that use the balanced encoding strategy and online image

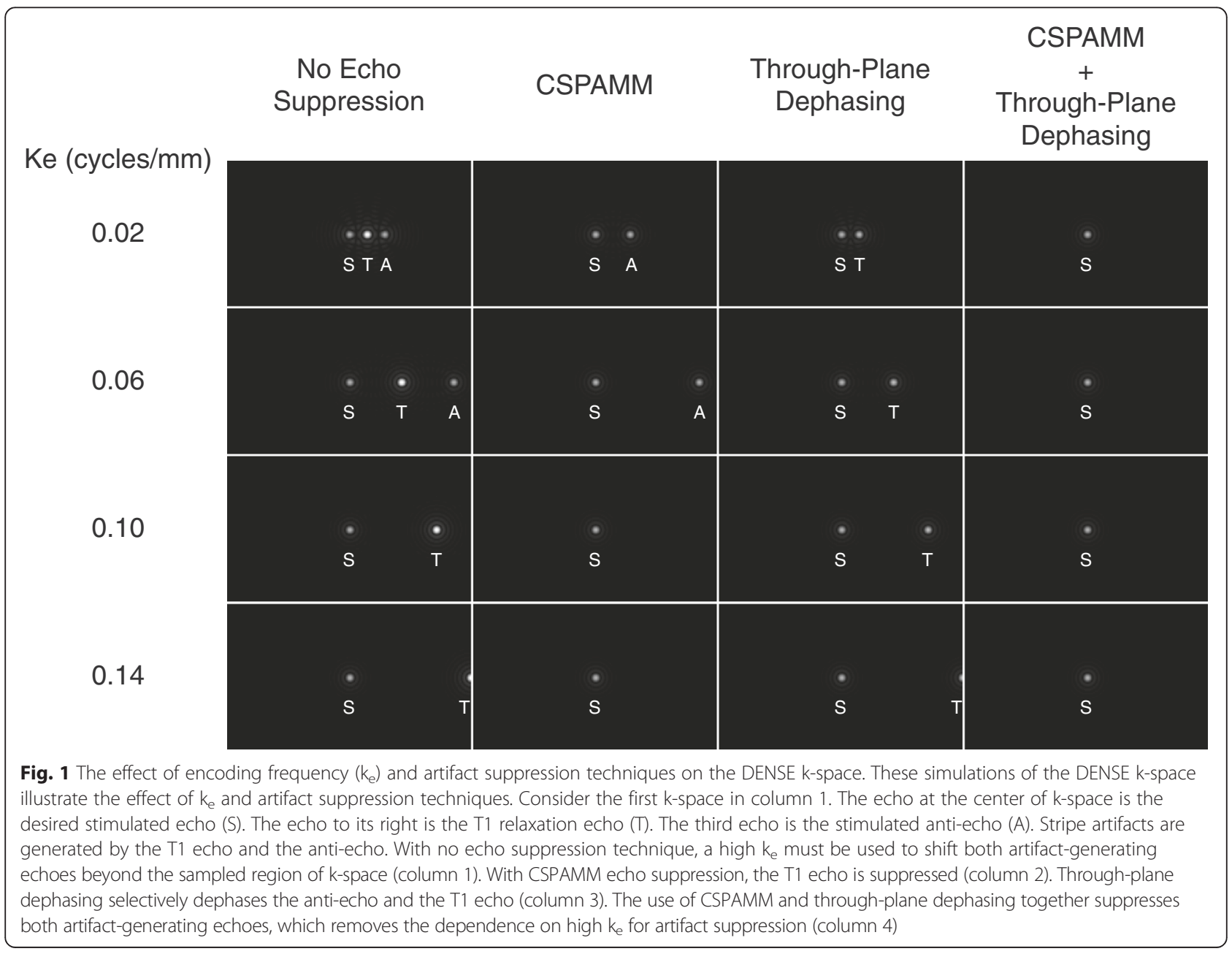


reconstruction suffer from up to three-fold increased phase wrapping [11] that may not be correctly resolved by the unwrapping algorithm, particularly in the presence of noise. Indeed, lower $k_{e}(0.06$ cycles $/ \mathrm{mm})$ have been used in these studies to reduce the amount of wrapping and simplify the input to unwrapping algorithms [11-13]. No direct comparisons with higher $k_{\mathrm{e}}$ have been performed to validate this approach.

Very low $\mathrm{k}_{\mathrm{e}}$ may be undesirable due to low sensitivity to displacement $[10,11]$. If the sensitivity is too low, there may be errors in the quantifications of cardiac mechanics. While this may be problematic as the $\mathrm{k}_{\mathrm{e}}$ approaches zero, a relatively low $k_{e}$ of 0.04 cycles $/ \mathrm{mm}$ is still able to resolve displacements of $0.006 \mathrm{~mm}$ with typical 12-bit data storage. More importantly, though, the sensitivity of the displacement measurements to phase noise increases with decreasing $k_{\mathrm{e}}$. No study has investigated a range of $k_{\mathrm{e}}$ to ascertain its effects on quantifications of cardiac mechanics. It has also been suggested that a high $\mathrm{k}_{\mathrm{e}}$ is required to dephase the blood pool signal [10]. This may not be the case, however, as long as a through-plane dephasing gradient is in place to accomplish the dephasing.

We hypothesized that 1) quantifications of myocardial circumferential strain, radial strain, and twist will not be different for encoding frequencies between 0.02 and 0.10 cycles $/ \mathrm{mm}, 2$ ) the nulling of the blood signal will be similar for all encoding frequencies, 3) the use of lower encoding frequencies will prevent phase wrapping even in healthy subjects with substantial cardiac motion, and 4) lower encoding frequencies will have higher SNR. We tested these hypotheses using a spiral cine DENSE protocol implemented on a $3 \mathrm{~T}$ Siemens Tim Trio MRI scanner.

\section{Methods}

\section{Image acquisition}

This protocol was approved by the local Institutional Review Board of the University of Kentucky. Ten healthy subjects (50\% female, age $27 \pm 9$ ) with no history of cardiovascular disease and ten subjects with a history of myocardial infarction or congestive heart failure ( $40 \%$ female, age $57 \pm 6$ ) consented for the study. A $3 \mathrm{~T}$ Siemens (Erlangen, Germany) Tim Trio with a 6-element chest and 24-element spine coil was used to acquire mid-ventricular short-axis 2D cine DENSE images with the following parameters: 6 spiral interleaves, 1 average, $360 \times 360 \mathrm{~mm}^{2}$ field of view, $128 \times 128$ reconstruction matrix, $2.8 \times$ $2.8 \mathrm{~mm}^{2}$ pixel size, $8 \mathrm{~mm}$ slice thickness, $1.08 \mathrm{~ms} / 17 \mathrm{~ms}$ $\mathrm{TE} / \mathrm{TR}$, constant $20^{\circ}$ flip angle. Two spirals were acquired per heartbeat which yielded a temporal resolution of $34 \mathrm{~ms}$. View sharing was used to achieve $17 \mathrm{~ms}$ between reconstructed cardiac frames. Simple encoding was used to measure in-plane displacements while through-plane dephasing of 0.08 cycles $/ \mathrm{mm}$ and CSPAMM were used for echo suppression $[3,7,11]$. To remove effects due to variable breath-hold position, the acquisitions were performed with respiratory navigator gating and an acceptance window of $\pm 3 \mathrm{~mm}$.

In each subject, the same mid-ventricular short-axis slice was acquired five times with different values of inplane $\mathrm{k}_{\mathrm{e}}: 0.02,0.04,0.06,0.08$, and 0.10 cycles $/ \mathrm{mm}$. The 0.10 cycles $/ \mathrm{mm}$ acquisition was repeated during the same imaging session to assess inter-test reproducibility.

\section{DENSE strain and twist analyses}

Myocardial strain and twist were derived from the DENSE images using custom software written in MATLAB (The Mathworks Inc, Natick, MA). The post-processing steps for each cine DENSE slice included manual segmentation of the left ventricular myocardium and semi-automated phase unwrapping to obtain the 2D Eulerian displacements within each cardiac frame [10]. Following the unwrapping, spatial smoothing and temporal fitting of displacements $\left(10^{\text {th }}\right.$ order polynomial) were performed to obtain smooth trajectories for all tissue points beginning at end-diastole and continuing through systole into much of diastole [10]. Radial strain, circumferential strain, and twist were calculated from the resulting displacement fields for each cardiac frame [14].

Radial and circumferential strains were quantified with the 2D Lagrangian finite strain tensor in six circumferential segments throughout the cardiac cycle. Radial strain was defined as positive for thickening while circumferential strain was negative for shortening. To report peak global strains, the curves from the six segments were averaged into a single global curve from which the peak was selected. Twist was quantified in the same segments and was defined as the angle of rotation about the centroid of the endocardial contour at end-diastole. Twist was positive for counterclockwise rotation when viewing the short-axis slice from the apex towards the base. Peak global twist was quantified in the same manner as the peak global strains.

As many recent studies have used a $\mathrm{k}_{\mathrm{e}}$ of 0.10 cycles $/ \mathrm{mm}$, the peak strains and twists quantified with the other $k_{e}$ were compared to the same measures quantified with a $k_{e}$ of 0.10 cycles $/ \mathrm{mm}$. Paired t-tests (with significance defined as $p<0.05$ ), Bland-Altman analyses [15], and modified coefficients of variation $(\mathrm{CoV})$ were used for statistical comparison. The equation for $\mathrm{CoV}$ is below for a given measurement, $X$, quantified in $N$ subjects with two encoding frequencies (ke1 and ke2) $[6,16]$.

$$
\operatorname{CoV}=\frac{\sum_{i=1}^{N}\left[\text { St.Dev. }\left(X_{k e 1} X_{k e 2}\right)_{i}\right] / N}{\left|\sum_{i=1}^{N}\left[\left(\left(X_{k e 1}+X_{k e 2}\right) / 2\right)_{i}\right] / N\right|}
$$

\section{Phase wrapping}

The amount of phase wrapping that occurred for a given subject and $k_{e}$ was measured by first considering the 
phase images for the $\mathrm{X}$ and $\mathrm{Y}$ directions separately. For each of the two directions, the cardiac frame with the largest percentage of wrapped pixels within the cardiac segmentation was found. The cardiac frame with this largest percentage may have been at slightly different time points for the two directions, though always near end-systole because that is when the most displacement and wrapping occurred. The average of those two percentages was taken as the amount of phase wrapping for that subject and $\mathrm{k}_{\mathrm{e}}$.

\section{Blood pool dephasing}

Dephasing of the blood signal through the cardiac cycle for each $k_{e}$ was quantified by calculating the average pixel intensity of the DENSE magnitude images within a set of manually defined contours that denoted the blood pool. Care was taken to ensure that the papillary muscles and trabeculations were not included within the blood pool for this analysis. The magnitude of the blood pool signal was quantified and expressed through the cardiac cycle as a percentage of its signal in the first cardiac phase. To demonstrate the amount of dephasing that has occurred by early systole, the blood pool signal remaining at the fifth cardiac frame $(85 \mathrm{~ms}$ into the cardiac cycle) was compared between the acquisitions with different $k_{\mathrm{e}}$

\section{Signal to noise ratio}

To compare the effects of intra-voxel dephasing between the different $k_{e}$, the signal to noise ratio (SNR) was calculated for each cardiac phase. The end-systolic SNR for each lower $k_{e}$ was compared to the SNR for $k_{e}$ of 0.10 cycles $/ \mathrm{mm}$ with a paired $t$-test. SNR was calculated from the magnitude images by finding the average signal within the myocardium and the standard deviation (noise) of signal within a region of zero signal outside of the body. Care was taken to avoid image artifacts in the region of zero signal. Corrections were applied for the Rician distribution of the MR signal [17]. The true standard deviation of the signal, $\sigma$, was calculated from the measured standard deviation, $\sigma_{\mathrm{M}}$, by

$$
\sigma=\sqrt{\frac{2}{4-\pi}} * \sigma_{M} \approx 1.526 * \sigma_{M}
$$

The true myocardial signal, $S$, was calculated from the measured myocardial signal, $M$, by

$$
S=\sqrt{M^{2}-\sigma^{2}}
$$

SNR was calculated as the ratio of $S$ to $\sigma$.

\section{Relationship between phase noise and SNR}

To assess the relationship between phase noise and SNR, the same DENSE acquisitions above were performed on a stationary water phantom. SNR was quantified in the same manner as for the human studies. For each $k_{e}$, the phase noise in the $X$ and $Y$ phase images was quantified via the root mean squared error (RMSE) in radians. To compute the RMSE of the 2D displacements, the previous RMSEs were converted from radians to millimeters via the $k_{\mathrm{e}}$. The $\mathrm{X}$ and Y RMSEs in millimeters were then added together via vector addition to yield the 2D RMSE. The phase noise in radians is theoretically inversely proportional to the SNR [17].

\section{Results}

As quantified by the DENSE acquisition with a $k_{e}$ of 0.10 cycles $/ \mathrm{mm}$, the patients had a mean ( \pm standard deviation) global circumferential strain of $-12 \pm 6 \%$ (range: -3 to $-20 \%$ ). The same measure in the healthy subjects was $-20 \pm 2 \%$ (range: -17 to $-23 \%$ ).

End-systolic images from a representative subject are shown in Fig. 2 and demonstrate a reduction in phase wrapping at lower $k_{\mathrm{e}}$. No phase wrapping was present within the segmentation of the myocardium for $k_{e}$ of 0.04 and 0.02 cycles $/ \mathrm{mm}$.

\section{DENSE strain and twist analyses}

Negligible differences were seen in strains and twist for all $\mathrm{k}_{\mathrm{e}}$ between 0.04 and 0.10 cycles/mm (Fig. 3, Table 1 ). These differences were of the same magnitude as intertest differences. The comparison between $k_{e}$ of 0.02 and 0.10 cycles $/ \mathrm{mm}$, however, demonstrated larger biases, larger $95 \%$ limits of agreement (LoA), and larger CoVs for both strains and twist. The differences in circumferential strain and twist between $\mathrm{k}_{\mathrm{e}}$ of 0.02 and 0.10 cycles $/ \mathrm{mm}$ were significant ( $p=<0.01$ and $p=0.04$, respectively).

\section{Phase wrapping}

For $\mathrm{k}_{\mathrm{e}}$ of $0.02,0.04,0.06,0.08$, and 0.10 cycles $/ \mathrm{mm}$, the largest percentage of wrapped pixels in the phase images was $0 \pm 0,0 \pm 0,5 \pm 6,17 \pm 10$, and $32 \pm 9 \%$, respectively. Thus, phase images acquired with a $\mathrm{k}_{\mathrm{e}}$ of 0.04 cycles/ $\mathrm{mm}$ had zero wrapped pixels. In contrast, the same phase images acquired with a $k_{e}$ of 0.10 cycles $/ \mathrm{mm}$ had about $32 \%$ of the pixels wrapped in the cardiac frame with the most displacement.

\section{Blood pool dephasing}

As $k_{e}$ increased, the rate of blood pool dephasing increased, however, the standard deviations demonstrated considerable overlap among the different $k_{e}$ (Fig. 4). Across the 20 subjects and using the fifth cardiac phase as an example, the amount of blood pool signal remaining as a percentage of its initial value was $28 \pm 11$, $26 \pm 10,24 \pm 9,23 \pm 8$, and $21 \pm 7 \%$ for $k_{\mathrm{e}}$ of $0.02,0.04$, $0.06,0.08$, and 0.10 cycles $/ \mathrm{mm}$, respectively. Frame 20 


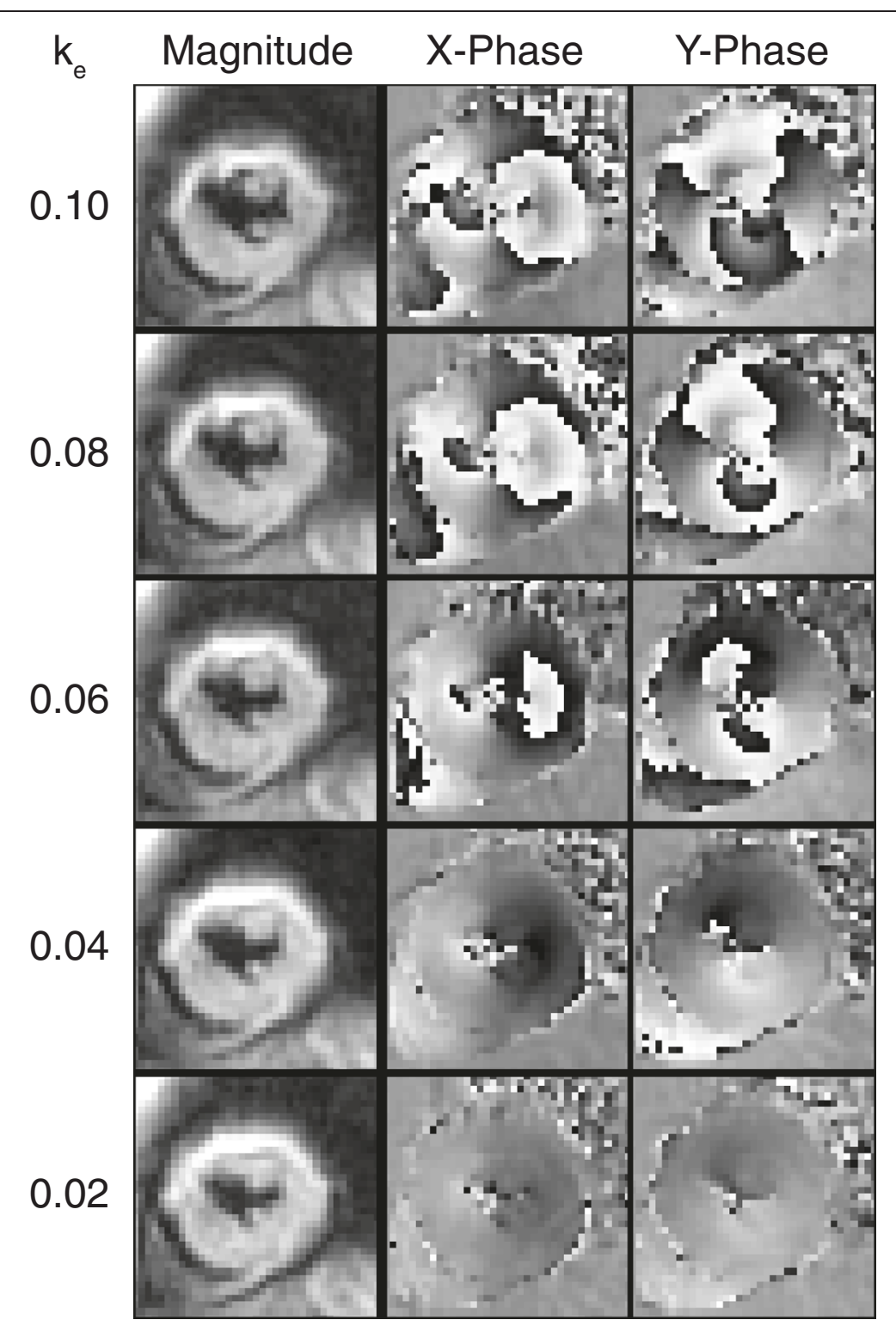

Fig. 2 End-systolic magnitude and phase images from a subject with previous myocardial infarction. Substantial wrapping was present in the phase images for the higher $k_{e}$. As the $k_{e}$ was decreased, the amount of wrapping in the $X$ and $Y$ phase images decreased. No wrapping was present in the myocardium for 0.02 and 0.04 cycles $/ \mathrm{mm}$. Also note that the blood pool dephased similarly for all $\mathrm{k}_{e}$

was the average end-systolic frame and there was no effective difference in blood pool dephasing by that time.

\section{Signal to noise ratio}

SNR throughout the cardiac cycle was similar for the different $\mathrm{k}_{\mathrm{e}}$ (Fig. 5), with a trend towards higher SNR at lower $\mathrm{k}_{\mathrm{e}}$. Across the 20 subjects, the mean SNR at endsystole, which occurred at different cardiac frames for the different subjects, was $23 \pm 9,24 \pm 9,23 \pm 9,23 \pm 10$, and $22 \pm 9$ for $\mathrm{k}_{\mathrm{e}}$ of $0.02,0.04,0.06,0.08$, and 0.10 cycles $/ \mathrm{mm}$, respectively. The end-systolic SNR for $k_{\mathrm{e}}=0.02,0.04,0.06$, and 0.08 were each significantly different than the endsystolic SNR for $\mathrm{k}_{\mathrm{e}}=0.10$ cycles $/ \mathrm{mm}(p=0.010,0.003$,
$0.005,0.03$, respectively). This represents a $9 \%$ increase in SNR for $k_{e}$ of 0.04 cycles $/ \mathrm{mm}$ compared to a $k_{e}$ of 0.10 cycles $/ \mathrm{mm}$.

\section{Relationship between phase noise and SNR}

In the stationary water phantom, the inverse relationship between the phase noise (as measured by RMSE in radians) and the SNR was similar for all $k_{\mathrm{e}}$ (Fig. 6a). However, the RMSE in millimeters, which required division by the appropriate $k_{e}$, was substantially higher for lower $k_{e}$ (Fig. $6 b$ ). For example, for SNR near 20, the RMSEs in millimeters were $1.17,0.60,0.38,0.30$, and $0.23 \mathrm{~mm}$, for $\mathrm{k}_{\mathrm{e}}=0.02,0.04$, $0.06,0.08$, and 0.10 cycles $/ \mathrm{mm}$, respectively. 


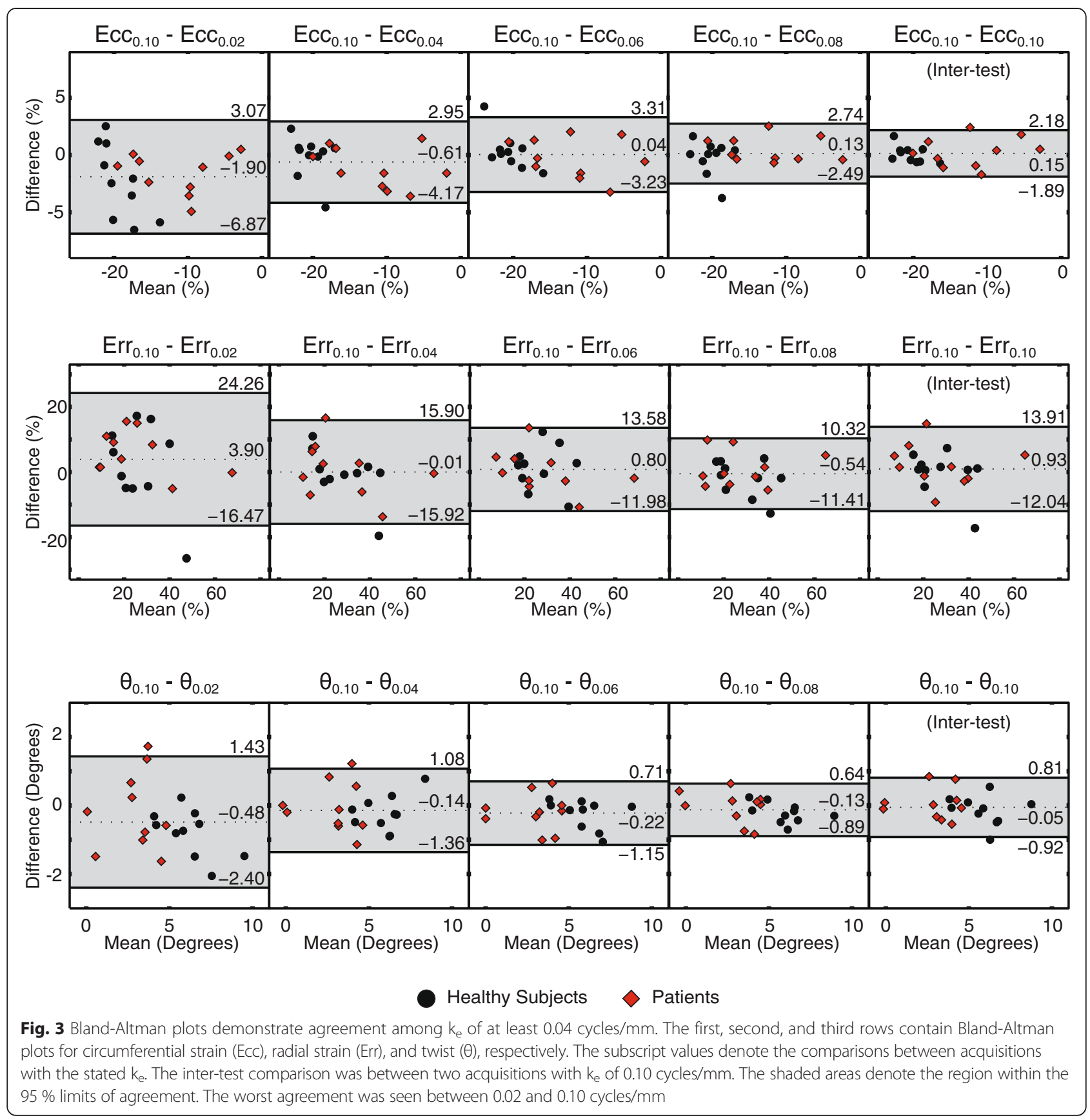

\section{Discussion}

Spiral 2D cine DENSE has typically been acquired with a $\mathrm{k}_{\mathrm{e}}$ of 0.10 cycles $/ \mathrm{mm}[5,8,9]$. This value is high enough to cause phase wrapping after only $5 \mathrm{~mm}$ of tissue displacement. In the present study, we investigated the hypothesis that lower $k_{e}$ could be used to reduce the amount of phase wrapping without compromising the quantification of strain and twist from midventricular short-axis images. Our primary findings included: 1) the $\mathrm{k}_{\mathrm{e}}$ can be reduced to 0.04 cycles $/ \mathrm{mm}$ without causing differences in the quantifications of circumferential strain, radial strain, or twist; 2) phase wrapping can be eliminated from the phase images with the use of $k_{e}$ less than or equal to 0.04 cycles $/ \mathrm{mm}$; 3 ) the rate of blood pool dephasing, which is a source of contrast between blood and myocardium in the magnitude images, is similar for $\mathrm{k}_{\mathrm{e}}$ between 0.02 and 0.10 cycles/mm; and 4) the SNR at end-systole is $9 \%$ higher when using a $\mathrm{k}_{\mathrm{e}}$ of 0.04 cycles $/ \mathrm{mm}$ compared to using a $\mathrm{k}_{\mathrm{e}}$ of 0.10 cycles $/ \mathrm{mm}$. 
Table 1 Summary statistics showed good agreement for all $k_{e}$ between 0.04 and 0.10 cycles/mm. Larger biases, 95 \% LoA, and CoVs were observed for $\mathrm{k}_{\mathrm{e}}$ of 0.02 cycles $/ \mathrm{mm}$

\begin{tabular}{|c|c|c|c|c|c|c|c|c|c|c|c|c|}
\hline \multirow[t]{2}{*}{$E\left(k_{e}\right)^{* *}$} & \multicolumn{4}{|c|}{ Circumferential Strain (\%) } & \multicolumn{4}{|c|}{ Radial Strain (\%) } & \multicolumn{4}{|c|}{ Twist (Degrees) } \\
\hline & Bias & $95 \%$ LoA & Mean CoV & $p$-value & Bias & $95 \%$ LoA & Mean CoV & $p$-value & Bias & $95 \%$ LOA & Mean CoV & $p$-value \\
\hline$E_{0.10}-E_{0.02}$ & -1.9 & \pm 5.0 & $11 \%$ & $<0.01^{*}$ & 3.9 & \pm 20.4 & $23 \%$ & 0.11 & -0.48 & \pm 1.92 & $14 \%$ & $0.04^{*}$ \\
\hline$E_{0.10}-E_{0.04}$ & -0.6 & \pm 3.6 & $6 \%$ & 0.15 & -0.0 & \pm 15.9 & $14 \%$ & 1.00 & -0.14 & \pm 1.22 & $8 \%$ & 0.32 \\
\hline$E_{0.10}-E_{0.06}$ & 0.0 & \pm 3.2 & $6 \%$ & 0.91 & 0.8 & \pm 12.8 & $13 \%$ & 0.59 & -0.22 & \pm 0.93 & $6 \%$ & 0.05 \\
\hline$E_{0.10}-E_{0.08}$ & 0.1 & \pm 2.6 & $4 \%$ & 0.67 & -0.5 & \pm 10.9 & $11 \%$ & 0.67 & -0.13 & \pm 0.77 & $5 \%$ & 0.16 \\
\hline Inter-test & 0.1 & \pm 2.0 & $4 \%$ & 0.53 & 0.9 & \pm 13.0 & $12 \%$ & 0.54 & -0.05 & \pm 0.87 & $5 \%$ & 0.59 \\
\hline
\end{tabular}

* Statistical significance between peak measures of mechanics using paired-sample $t$-test at significance level $a=0.05$

${ }^{*} E_{\left[\boldsymbol{k}_{\boldsymbol{e}}\right]}$ represents peak strain or twist measured using a particular $\mathrm{k}_{\mathrm{e}}$

Abbreviations: LoA limits of agreement, CoV coefficient of variation

\section{DENSE strain and twist analyses}

Spiral cine DENSE is primarily used to measure cardiac displacements and deformation in the forms of twist and strain $[3,10,12]$. The $k_{\mathrm{e}}$ is the proportionality constant between the tissue displacement in millimeters and the measured signal phase. It also determines the strength of the encoding gradient that is applied. A high value of $\mathrm{k}_{\mathrm{e}}$ provides high sensitivity to small displacements, but at the cost of intra-voxel dephasing and increased phase wrapping. The results from this study suggest that the $\mathrm{k}_{\mathrm{e}}$ can be lowered to 0.04 cycles $/ \mathrm{mm}$, which significantly reduces the presence of phase wrapping, without compromising measures of circumferential strain, radial strain, or twist. In addition, studies that use different $\mathrm{k}_{\mathrm{e}}$ between 0.04 and 0.10 cycles $/ \mathrm{mm}$ can be directly compared as no systematic differences in strain or twist due to differences in $k_{e}$ were found. This is valuable as not all DENSE studies have used the typical value of
0.10 cycles $/ \mathrm{mm}$. In particular, some previous studies have used 0.06 cycles/mm $[12,13]$, which is within the range of this study.

The measures of strain and twist were compromised as the $\mathrm{k}_{\mathrm{e}}$ was lowered to 0.02 cycles $/ \mathrm{mm}$ (Fig. 3). This was likely caused by the increased effect of phase errors at low $\mathrm{k}_{\mathrm{e}}$ (Fig. 6b). For a given phase error in radians, the corresponding error in displacement $(\mathrm{mm})$ was larger for lower $k_{\mathrm{e}}$. This same phenomenon is present in phase contrast velocity imaging as the velocity encoding (VENC) is increased [18].

\section{Phase wrapping}

The amount of phase wrapping decreased as the $k_{e}$ was decreased. Lowering the $k_{\mathrm{e}}$ to the point that there is no wrapping puts DENSE on a similar level as phase contrast velocity imaging, where the VENC is commonly adjusted to prevent wrapping in the blood velocities [19].

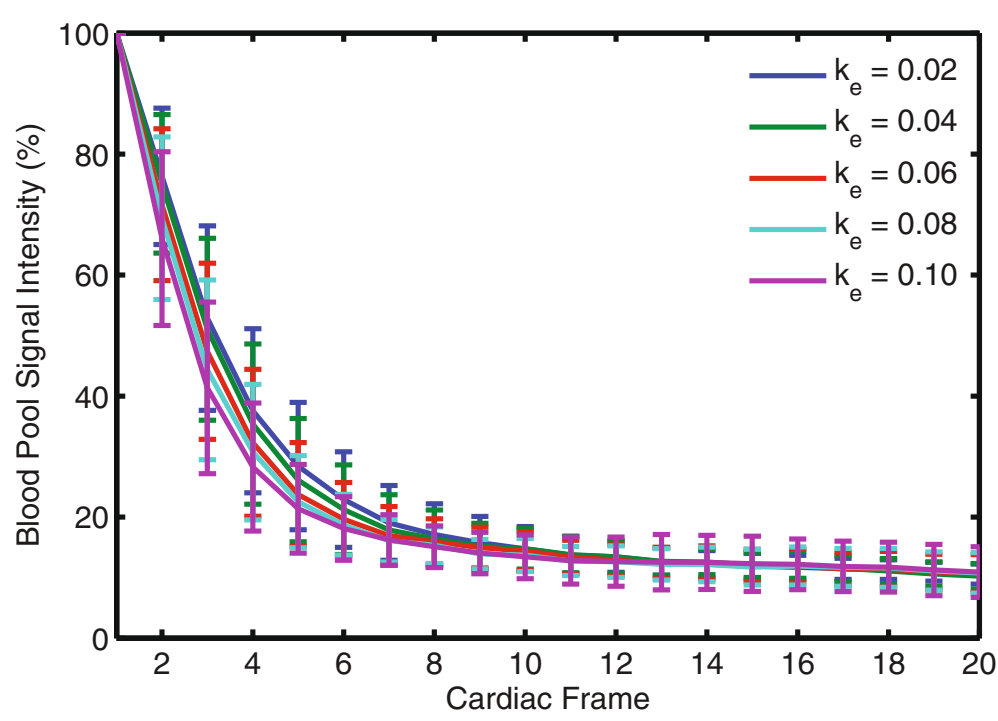

Fig. 4 Similar rates of blood pool dephasing were observed for the different $k_{e}$. Blood pool signal intensity was expressed as a percentage of its value at the first cardiac phase. The first 20 cardiac frames are shown. Each curve represents the average of the 20 subjects with standard deviation error bars. As the $k_{e}$ increased, the rate of blood pool dephasing increased, but with considerable overlap between the different $k_{e}$ as seen by the wide standard deviation bars 


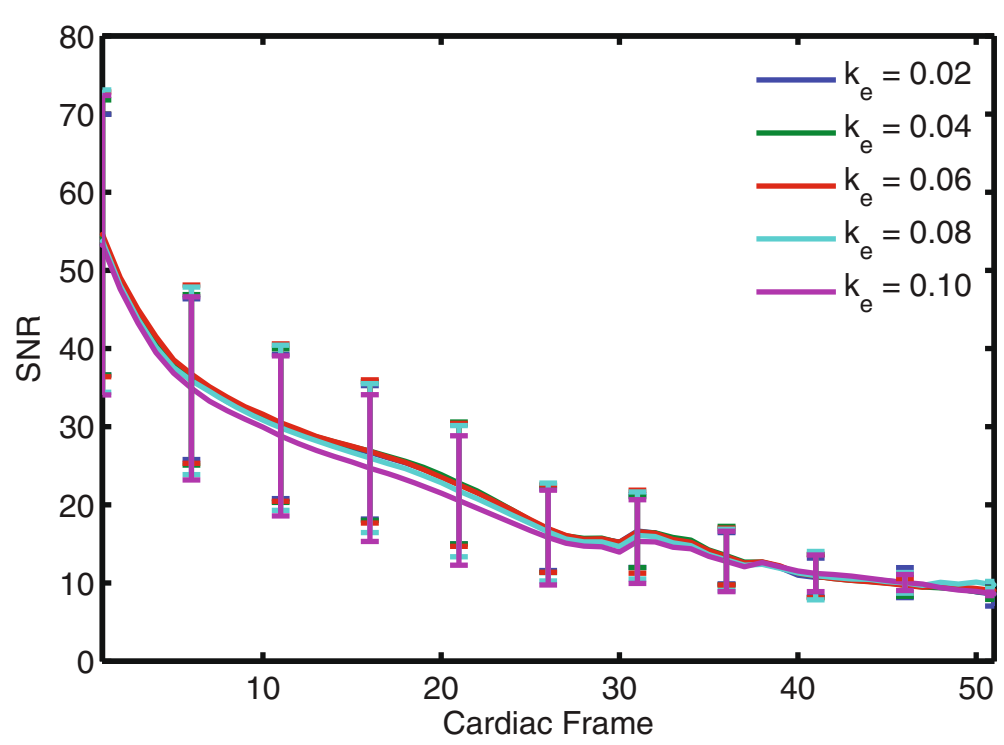

Fig. 5 The SNR throughout the cardiac cycle was similar for the different $k_{\mathrm{e}}$. Each curve represents the average of the 20 subjects with standard deviation error bars. Starting with the first frame, the standard deviation is shown at every fifth cardiac frame for clarity. There is a trend towards higher SNR at lower $k_{e}$, particularly between the $15^{\text {th }}$ and $20^{\text {th }}$ cardiac frames

The use of this low value was possible due to the artifact suppression techniques of CSPAMM [3] and throughplane dephasing [7]. As seen in the representative subject (Fig. 2), no stripe artifacts were present in the images for the low $\mathrm{k}_{\mathrm{e}}$.

\section{Blood pool dephasing}

The rate of blood pool dephasing decreased as the $k_{e}$ was decreased (Fig. 4). However, the difference between the acquisitions with 0.10 and 0.02 cycles $/ \mathrm{mm}$ was not large. By the fifth cardiac frame, the acquisition with 0.02 cycles $/ \mathrm{mm}$ had approximately $7 \%$ more of its blood pool signal remaining. This difference was not practically significant as the delineation between the myocardium and the blood pool was still possible at the lowest $\mathrm{k}_{\mathrm{e}}$. The drop in blood pool signal through the cardiac cycle is due to dephasing [10]. This dephasing can be due to both in-plane and through-plane gradients. While the in-plane gradients necessarily changed with the $\mathrm{k}_{\mathrm{e}}$, the through-plane gradient remained constant for all acquisitions and likely contributed to the blood pool dephasing at similar rates for all $\mathrm{k}_{\mathrm{e}}$. Thus, the advent of through-plane dephasing removed dependence on high $\mathrm{k}_{\mathrm{e}}$ to accomplish blood pool dephasing.
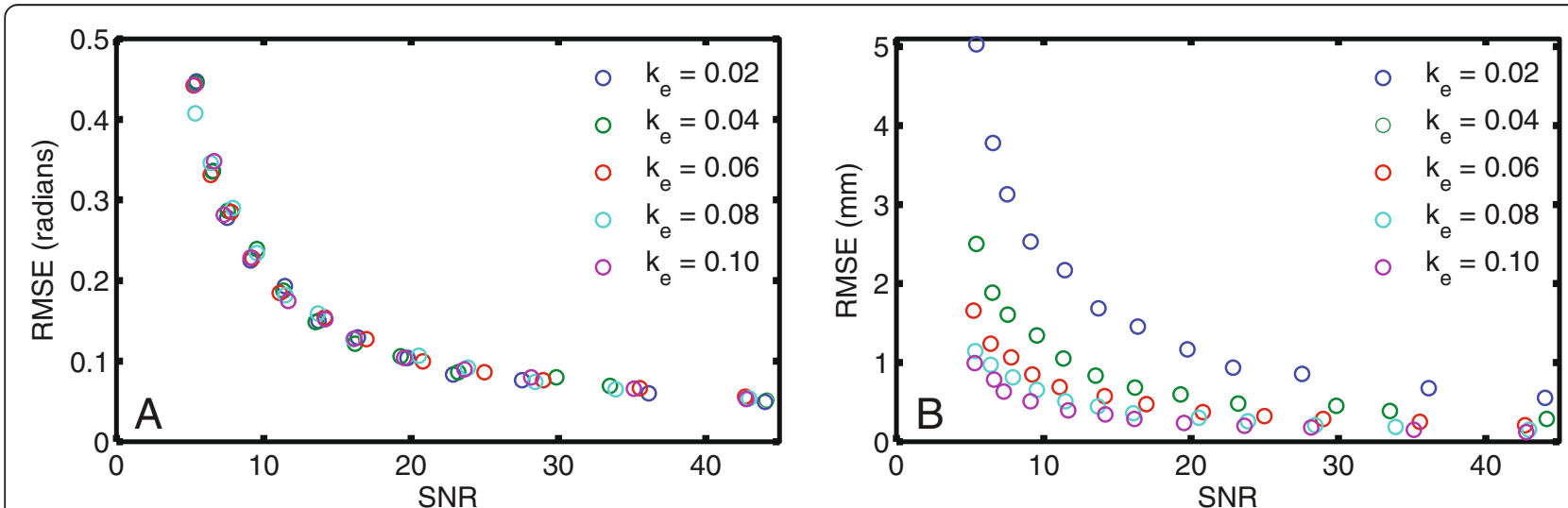

Fig. 6 Phase noise had a larger effect on displacement errors with lower $k_{\text {e. }}$ a In a stationary water phantom, phase noise, as quantified by RMSE in radians, was inversely related to SNR. No differences in RMSE were seen between the different $k_{e}$. b When RMSE in radians was converted to millimeters by dividing by $k_{e}$, there were substantial differences between the different $k_{e}$. Lower $k_{e}$ had increased displacement errors 


\section{Signal to noise ratio}

The SNR was $9 \%$ higher for $\mathrm{k}_{\mathrm{e}}$ of 0.04 cycles $/ \mathrm{mm}$ compared to 0.10 cycles $/ \mathrm{mm}$. This is a reflection of the decreased intra-voxel dephasing that occurs due to the decreased gradient strengths that accompany lower $\mathrm{k}_{\mathrm{e}}$. This modest increase in SNR is generally beneficial and results in a reduction in phase noise [17].

It is important to note that both the in-plane encoding gradient and the through-plane dephasing gradient are capable of producing intra-voxel dephasing of the stimulated echo in deforming tissue [7]. The voxel size in the through-plane direction was larger than the in-plane direction $(8 \mathrm{~mm}$ vs. $2.8 \mathrm{~mm}$ ). Thus, the amount of intravoxel dephasing may have been largely controlled by the through-plane dephasing gradient, which was constant $(0.08$ cycles $/ \mathrm{mm})$ for all acquisitions in this study. Further increases in SNR could be possible by reducing the through-plane dephasing gradient, however, this value was chosen to cause more than one half cycle of dephasing across the $8 \mathrm{~mm}$ slice [7]. Reducing the amount of through-plane dephasing could lead to the presence of stripe artifacts in the images.

\section{Limitations}

This study assessed a single mid-ventricular short-axis slice without consideration of long-axis images. The longitudinal motion of the left ventricle (particularly near the base) is often larger than the circumferential and radial components [20]. Long-axis images would likely have demonstrated phase wrapping with a $\mathrm{k}_{\mathrm{e}}$ of 0.04 cycles $/ \mathrm{mm}$. While this implies that unwrapping algorithms cannot be removed from the post-processing, the amount of wrapping can be substantially reduced with a lower value. As the circumferential and radial strains were not compromised in the short-axis images with this low value, the longitudinal strains from the long-axis images should also not be compromised.

The acquisitions in this study were performed at $3 \mathrm{~T}$, which yields higher SNR compared to $1.5 \mathrm{~T}$ [21]. Acquisitions at $1.5 \mathrm{~T}$ may have larger phase errors (due to decreased SNR) than those present in this study. However, those errors could be offset by better field homogeneity at the lower field strength. It has recently been reported that the displacement errors from spiral cine DENSE are the same at $3 \mathrm{~T}$ and $1.5 \mathrm{~T}$ [16]. Thus, the results from this study are likely applicable to $1.5 \mathrm{~T}$.

We performed the acquisitions in this study with the simple encoding strategy because of the reported ability to handle phase wrapping due to $\mathrm{k}_{\mathrm{e}}$ as high as $0.10 \mathrm{cy}$ cles/mm [10]. A motivation for this study, however, was to investigate the ability to lower the $\mathrm{k}_{\mathrm{e}}$ during acquisitions that use the balanced encoding strategy. This strategy has been used for DENSE acquisitions that encode displacements in all three directions [11-13]. However, in those studies, the $\mathrm{k}_{\mathrm{e}}$ was reduced to 0.06 cycles $/ \mathrm{mm}$ due to the increased wrapping that is present in the online reconstructed images [11]. We could not guarantee successful unwrapping from images acquired with the balanced strategy and a $k_{e}$ of 0.10 cycles $/ \mathrm{mm}$, so the simple strategy was used to be able to accurately test up to 0.10 cycles $/ \mathrm{mm}$. The results from this study suggest that the $\mathrm{k}_{\mathrm{e}}$ could likely be lowered to 0.04 cycles $/ \mathrm{mm}$ with the balanced strategy, which has better noise performance than the simple encoding strategy [11]. This lower value would reduce the load on the unwrapping algorithm for 3D DENSE studies and any DENSE studies that use the balanced encoding strategy. The strain and twist results from this study suggest that these measures of cardiac mechanics would not be compromised with the lower value.

\section{Conclusions}

Cine DENSE is typically acquired with an encoding frequency of 0.10 cycles/mm [5, 8, 9]. This value allows for high sensitivity to tissue displacements, but at the cost of substantial phase wrapping. We demonstrated that the encoding frequency can be lowered to 0.04 cycles $/ \mathrm{mm}$ to nearly eliminate phase wrapping without compromising the quantification of cardiac strains or twist. Future studies may take advantage of this lower value to reduce the amount of wrapping and simplify the input to unwrapping algorithms. In addition, studies performed with different encoding frequencies between 0.04 and 0.10 cycles $/ \mathrm{mm}$ can be directly compared as there is no systematic bias.

\section{Abbreviations}

CMR: Cardiovascular magnetic resonance; CoV: Coefficient of variation; CSPAMM: Complementary spatial modulation of magnetization; DENSE: Displacement encoding with stimulated echoes; ke: Encoding frequency; MR: Magnetic resonance; RMSE: Root mean squared error; SNR: Signal to noise ratio; TE: Echo time; TR: Repetition time; VENC: Velocity encoding.

\section{Competing interests}

Dr. Xiaodong Zhong is a Siemens employee. No other authors have competing interests to disclose.

\section{Authors' contributions}

GW analyzed and collected data, assisted with study design and implementation, and drafted the manuscript. JG analyzed and collected data, assisted with implementation, and assisted with drafting the manuscript. JS assisted with study design and implementation, data analysis and interpretation, and critical revision of the manuscript. $\mathrm{CH}, \sqcup, \mathrm{DP}, \mathrm{SH}, \mathrm{MV}$, and $\mathrm{XZ}$ assisted with implementation and critical revision of the manuscript. BF conceived the study, assisted in study design and coordination, and assisted with drafting and critical revision of the manuscript. All authors read and approved the final manuscript.

\section{Acknowledgements}

This work was supported by a National Institutes of Health $(\mathrm{NIH})$ Director's Early Independence Award (DP5 OD-012132), NIH grant number T32 HL-072743, and $\mathrm{NIH}$ grant number UL1TR000117 from the National Center for Research Resources and the National Center for Advancing Translational Sciences. The content is solely the responsibility of the authors and does not necessarily represent the official views of $\mathrm{NIH}$. 


\section{Author details}

${ }^{1}$ Department of Biomedical Engineering, University of Kentucky, Lexington, KY, USA. ${ }^{2}$ Department of Pediatrics, University of Kentucky, Lexington, KY, USA. ${ }^{3}$ Department of Electrical Engineering, University of Kentucky, Lexington, KY, USA. ${ }^{4}$ MR R\&D Collaborations, Siemens Healthcare, Atlanta, GA, USA. 'Department of Physiology, University of Kentucky, Lexington, KY, USA. ${ }^{6}$ Department of Medicine, University of Kentucky, Lexington, KY, USA. ${ }^{7}$ Institute for Advanced Application, Geisinger Health System, Danville, PA, USA. ${ }^{8}$ Institute for Advanced Application, Geisinger Clinic, 100 North Academy Avenue, Danville, PA 17822-4400, USA.

Received: 1 May 2015 Accepted: 26 October 2015

Published online: 04 November 2015

\section{References}

1. Aletras AH, Ding S, Balaban RS, Wen H. DENSE: displacement encoding with stimulated echoes in cardiac functional MRI. J Magn Reson. 1999;137:247-52.

2. Aletras $A H$, Balaban RS, Wen $H$. High-resolution strain analysis of the human heart with fast-DENSE. J Magn Reson. 1999;140:41-57.

3. Kim D, Gilson WD, Kramer CM, Epstein FH. Myocardial tissue tracking with two-dimensional cine displacement-encoded MR imaging: development and initial evaluation. Radiology. 2004;230:862-71.

4. Ernande L, Thibault H, Bergerot C, Moulin P, Wen H, Derumeaux G, et al. Systolic myocardial dysfunction in patients with type 2 diabetes mellitus: identification at MR imaging with cine displacement encoding with stimulated echoes. Radiology. 2012;265:402-9.

5. Bilchick KC, Kuruvilla S, Hamirani YS, Ramachandran R, Clarke SA, Parker KM, et al. Impact of mechanical activation, scar, and electrical timing on cardiac resynchronization therapy response and clinical outcomes. J Am Coll Cardiol. 2014:63:1657-66.

6. Haggerty CM, Kramer SP, Binkley CM, Powell DK, Mattingly AC, Charnigo R, et al. Reproducibility of cine displacement encoding with stimulated echoes (DENSE) cardiovascular magnetic resonance for measuring left ventricular strains, torsion, and synchrony in mice. J Cardiovasc Magn Reson. 2013;15:71.

7. Zhong X, Spottiswoode BS, Cowart EA, Gilson WD, Epstein FH. Selective suppression of artifact-generating echoes in cine DENSE using throughplane dephasing. Magn Reson Med. 2006;56:1126-31.

8. Young AA, Li B, Kirton RS, Cowan BR. Generalized spatiotemporal myocardial strain analysis for DENSE and SPAMM imaging. Magn Reson Med. 2012:67:1590-9.

9. Budge LP, Helms AS, Salerno M, Kramer CM, Epstein FH, Bilchick KC. MR cine DENSE dyssynchrony parameters for the evaluation of heart failure: comparison with myocardial tissue tagging. JACC Cardiovasc Imaging. 2012;5:789-97.

10. Spottiswoode BS, Zhong $X$, Hess AT, Kramer CM, Meintjes EM, Mayosi BM, et al. Tracking myocardial motion from cine DENSE images using spatiotemporal phase unwrapping and temporal fitting. IEEE Trans Med Imaging. 2007;26:15-30.

11. Zhong X, Helm PA, Epstein FH. Balanced multipoint displacement encoding for DENSE MRI. Magn Reson Med. 2009;61:981-8.

12. Zhong X, Spottiswoode BS, Meyer CH, Kramer CM, Epstein FH. Imaging three-dimensional myocardial mechanics using navigator-gated volumetric spiral cine DENSE MRI. Magn Reson Med. 2010;64:1089-97.

13. Auger DA, Zhong X, Epstein FH, Spottiswoode BS. Mapping right ventricular myocardial mechanics using 3D cine DENSE cardiovascular magnetic resonance. J Cardiovasc Magn Reson. 2012;14:4.

14. Zhong X, Gibberman LB, Spottiswoode BS, Gilliam AD, Meyer CH, French $\mathrm{BA}$, et al. Comprehensive cardiovascular magnetic resonance of myocardial mechanics in mice using three-dimensional cine DENSE. J Cardiovasc Magn Reson. 2011;13:83.

15. Bland JM, Altman DG. Statistical methods for assessing agreement between two methods of clinical measurement. Lancet. 1986;1:307-10.

16. Wehner GJ, Suever JD, Haggerty CM, Jing L, Powell DK, Hamlet SM, et al. Validation of in vivo 2D displacements from spiral cine DENSE at 3T. J Cardiovasc Magn Reson. 2015;17:1-11.

17. Gudbjartsson H, Patz S. The Rician distribution of noisy MRI data. Magn Reson Med. 1995:34:910-4.

18. Pelc NJ, Sommer FG, Li KC, Brosnan TJ, Herfkens RJ, Enzmann DR. Quantitative magnetic resonance flow imaging. Magn Reson Q. 1994;10:125-47.
19. Nett EJ, Johnson KM, Frydrychowicz A, Del Rio AM, Schrauben E, Francois CJ, et al. Four-dimensional phase contrast MRI with accelerated dual velocity encoding. J Magn Reson Imaging. 2012;35:1462-71.

20. Moore CC, Lugo-Olivieri CH, McVeigh ER, Zerhouni EA. Three-dimensional systolic strain patterns in the normal human left ventricle: characterization with tagged MR imaging. Radiology. 2000;214:453-66.

21. Sigfridsson A, Haraldsson $H$, Ebbers T, Knutsson $H$, Sakuma $H$. In vivo SNR in DENSE MRI; temporal and regional effects of field strength, receiver coil sensitivity and flip angle strategies. Magn Reson Imaging. 2011;29:202-8.

\section{Submit your next manuscript to BioMed Central and take full advantage of:}

- Convenient online submission

- Thorough peer review

- No space constraints or color figure charges

- Immediate publication on acceptance

- Inclusion in PubMed, CAS, Scopus and Google Scholar

- Research which is freely available for redistribution

Submit your manuscript at www.biomedcentral.com/submit
C Biomed Central 